\title{
Astrobiology with Robotic Telescopes at CAB
}

\section{Luis Cuesta, M. Teresa Eibe, Aurora Ullán, Antonio Pérez-Verde, and Jorge Navas}

\author{
Laboratorio de Astrofísica Estelar y Exoplanetas, Centro de Astrobiología, Torrejón de Ardoz, 28850 Madrid, Spain
}

Correspondence should be addressed to Luis Cuesta, cuestacl@inta.es

Received 30 June 2009; Accepted 30 October 2009

Academic Editor: Alberto J. Castro-Tirado

Copyright (C) 2010 Luis Cuesta et al. This is an open access article distributed under the Creative Commons Attribution License, which permits unrestricted use, distribution, and reproduction in any medium, provided the original work is properly cited.

\begin{abstract}
The key objectives of RTRCAB are the identification of new exoplanets and especially the characterization of the known exoplanets by observing photometric and systematic monitoring of their transits. These telescopes, equipped with advanced technology, optimized control programs, and optical and technical characteristics adequate for this purpose, are ideal to make the observations that are required to carry out these programs. The achievement of these goals is ensured by the existence of three separated geographical stations. In this sense, there are several planned missions that have the same objectives among their scientific goals, like Kepler, CoRoT, GAIA, and PLATO.
\end{abstract}

\section{Introduction}

Robotic telescopes are new tools that are changing many aspects of the way we do astronomy. The use of this type of telescope has been quickly adopted in some of the most prolific fields of this science, including searches for exoplanets, see Figure 1. In this particular field, robotic telescopes have demonstrated an efficiency and agility that would hardly have been obtained with conventional telescopes.

Robotic telescopes are mostly destined to study nearby and brilliant objects but are perfect to support experiments that require constant monitoring of the sky. Also, robotic telescopes are very useful in routine observations that do not require an operator and for which a great amount of data is generated but can be reduced and analyzed automatically.

In addition to the typical scientific uses, robotic telescopes are being used as fundamental pieces in the practical education of astronomy. Numerous institutes and museums have begun to develop their own robotic telescopes to bring astronomy into the classroom, as a way to understand the Universe that surrounds us. By establishing collaborations between institutes in different countries observations can be made in the classroom without a change in school schedules, which has an enormous benefit to the education community.

\section{Science with Robotic Telescopes}

The study of exoplanets is in the vanguard of the present investigation of astrophysics and is of great interest to the field of astrobiology. The method of transit detection is based on obtaining differential photometry to detect changes in the light curves of stars $[1,2]$. For this type of study a great amount of observation time is required.

Robotic telescopes are very useful for discovering new bodies in the Solar System or tracking already discovered objects, in particular near Earth objects (NEOs) $[3,4]$, see Figure 2. The study of NEOs is fundamental to increasing our understanding of the rates of impacts on planets, something clearly linked to the origin and the evolution of life.

The study of stars similar to the Sun is also important (particularly in terms of astrobiological implications) and has benefited from the use of these telescopes. A study that covers a wide range of star ages and metallicities can provide data that allow us to determine their zones of habitability. Photometric analysis of these stars will connect the microvariability with the chromospheric and magnetic activities of our star.

Systematic observation projects cover an important part of the time of observation with robotic telescopes, such as search for extragalactic supernovae, astrometry of comets and asteroids, variable star, and binary eclipsing photometry. 


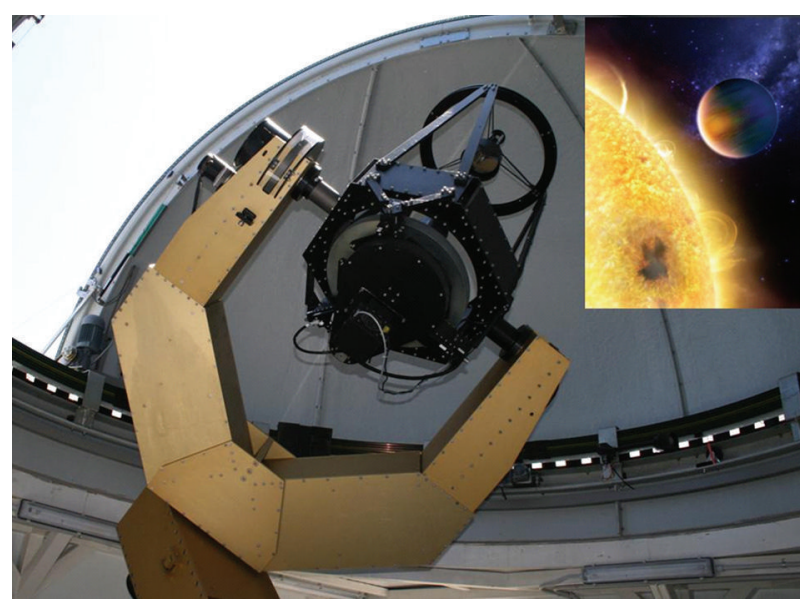

FIGURE 1: Robotic telescopes are one of the best tools for searching exoplanets.

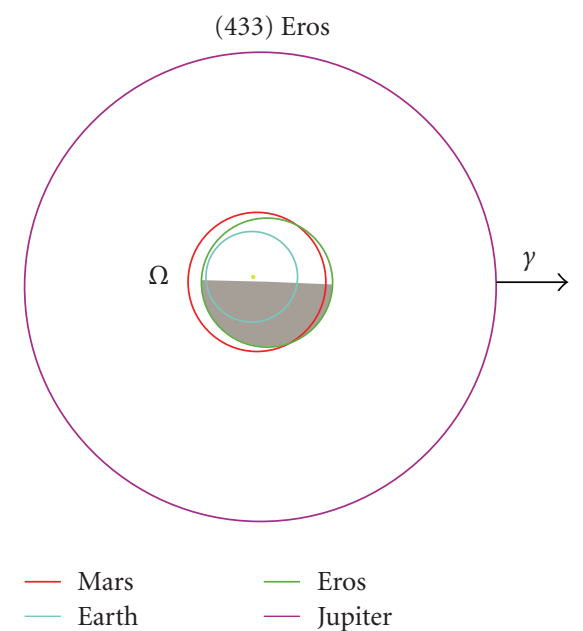

FIgURE 2: Orbit of asteroid Eros.

Also, time is dedicated to targets of opportunity like novae, supernovae, and gamma ray bursts.

\section{Exoplanets}

Since 1995, when the first exoplanet was discovered by Mayor and Queloz [5, 6], see Figure 3, and Marcy and Butler [7], more than 300 exoplanets (including dozens of multiplanets) have been found. Although a very interesting picture of this rich "zoology" is emerging [8-10], several important questions remain unanswered: what is the diversity of planetary systems, including long periods of planets and exotic systems? What is their dynamical evolution? What are the properties of exoplanets (orbits, masses, and properties of the atmosphere)? What are the frequency and properties of belt planetesimales and what are the implications for the frequency of formation of terrestrial planets, the habitability of planetary systems, and water distribution in the region of the terrestrial planets? How common are planets similar to Earth? These and other questions are of vital importance

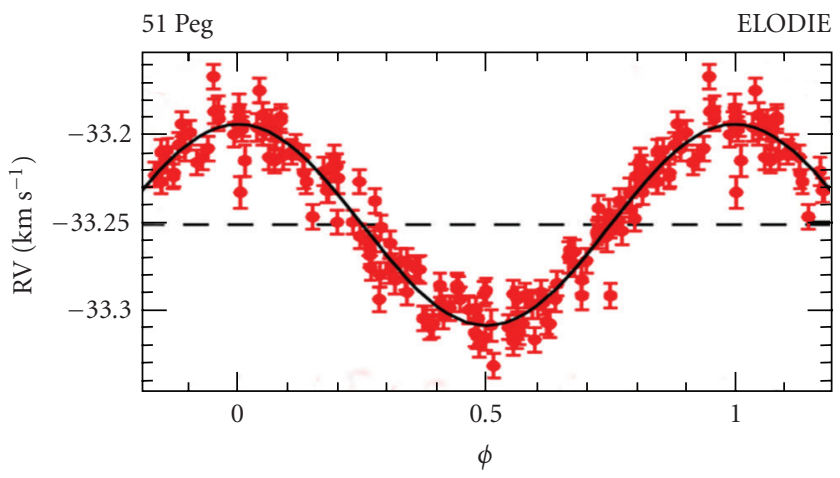

(a)

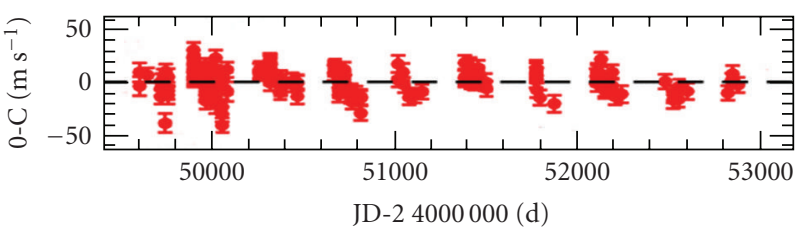

(b)

FIGURE 3: Radial velocity curve for 51 Peg where the first exoplanet was detected in 1995 by Mayor and Queloz $[5,6]$.

from an astrobiological point of view because they can help us to place the evolution of the Solar System and the establishment of basic habitability conditions on at least one of its planets, the Earth, into perspective. This project aims to answer some of these issues by addressing the problem from an observational approach and using this network of robotic telescopes.

During the last two decades there has been an intensive search for exoplanets, and as a result of this effort, there are now more than 300 exoplanets confirmed [11-13], most of them orbiting solar-type stars, with a mass of at least one quarter of the Neptune. Due to bias by instrumental observation techniques (most of the planets have been detected with the radial velocity method, with high-resolution spectrograph in the optical), there is not a census impartial of exoplanets or a full knowledge of their properties. In most cases the exoplanets found are giant and hot, usually found in very early stages of formation, and very close to its star. These properties are closely related to the observational bias introduced by the radial velocity method. The stars with known exoplanets are rich in metals compared to those that do not harbor planets. The number of stars with giant planets around seems to increase with metallicity of the star. For most of the exoplanets found we only know orbital parameters with large errors. Taking advantage of the potential use of the network of robotic telescopes, it is intended to study in depth the parameters of the known exoplanets to follow the curves of light and orbital cycles and phases of maximum and minimum so as to reduce drastically error bars. This will enable us to determine with greater precision the masses, sizes, and distances to the central star of the planets. Thus, we will select in a best approach those that might be within the habitable zone of the star. Also, such an 
observation would help to discriminate among the possible causes for the effect of the relationship between metallicity and the presence of planets in a star [14].

From an astrobiological point of view, the study of the atmosphere of exoplanets is of vital importance. The key element is the detection of which can be regarded as traces of the existence of life on earth terms. However, until now, there is not too much work on this topic and the only detections of exoplanet atmospheres have been made from space [15-18]. In the context of this project, we want to use the network of robotic telescopes to identify the presence of such atmospheres. The study is based on a novel analysis of the curves of light during the transit of the exoplanet, see Figure 4. Performing differential photometry during the rapid entry and exit of the transit it is possible to identify the atmosphere through a small change (around 1\%) occurred at the beginning and end of the depression in the light curve [19]. The analysis may reveal the presence of the atmosphere that would be subsequently studied in depth with other instruments.

Using the experience of the group in the optical observation and with the extensive existing database, it is expected to cover a substantial number of regions in order to produce a comprehensive census of the characteristics of exoplanets. Depending on the obtained results, we will apply the method of finding exoplanets by its transit to stars with evolutionary stages different of the Sun; for example, $M$ stars or planetary nebulae are ideal candidates for this search. In this last case, the knowledge of their nebular properties, as the inclination or the existence of disks of dust in the nuclear area, provides valuable information to establish in advance the possibility of a transit, if there is an exoplanet, and will help us to interpret much better light curves to establish the properties of the exoplanet.

\section{CAB's Robotic Telescopes}

The Spanish Instituto Nacional de Técnica Aeroespacial has a network of three telescopes in mainland Spain [20]. The first one is located at some of the best places for astronomy at the Observatorio de Calar Alto in Almería, at an altitude of more than $2100 \mathrm{~m}$, see Figure 5. The second one is near Calatayud in Zaragoza, at the summit of a $1400 \mathrm{~m}$ high mountain. The last one is on the campus of the Instituto Nacional de Técnica Aerospacial (INTA), in Madrid. The three telescopes have a classic Cassegrain configuration, with an aperture of 40$50 \mathrm{~cm}$ and motorized and automated equatorial mounts and domes.

Although the Calar Alto Observatory has a high rate of clear nights, the use of three telescopes drastically increases the chances of observation in case of bad weather. Moreover, this way makes possible the simultaneous observation with two telescopes for ultrafast photometry in different filters.

4.1. Mechanics and Optics. The three telescopes, built by Optical Mechanics Inc., are classic Cassegrain telescopes with a parabolic primary and a hyperbolic secondary mirror. The telescopes at Calar Alto and Calatayud both have a diameter

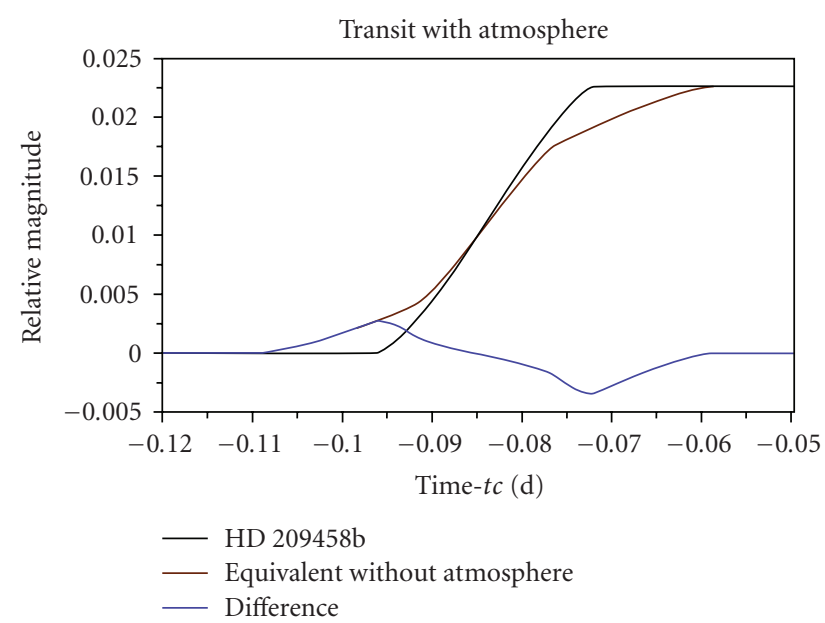

FIgURE 4: Theoretical effect of the presence of an atmosphere in the light curve of an exoplanet transit. The transit was used for HD 209458 b, with the slope equivalent to a planet with no atmosphere and the difference between both.

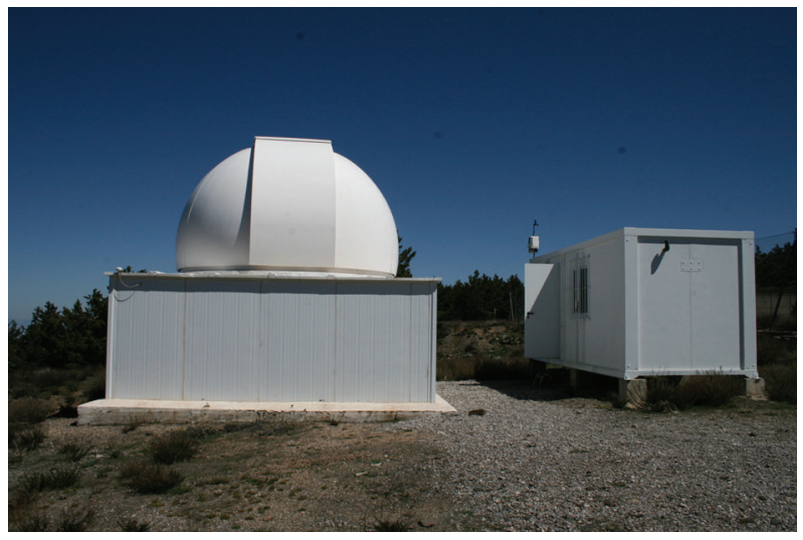

Figure 5: Observation station at Calar Alto Observatory. There is an auxiliary building with the wheater station and the control system.

of $50 \mathrm{~cm}$ while the third, at INTA, has a diameter of $40 \mathrm{~cm}$. All three telescopes are F/10.

The equatorial fork-mounted system is built from anodized aluminum. Right ascension and declination axes are driven by high-precision zero-lash drive systems. The relative pointing is less than 0.5 arcsecond and the final tracking resolution is less than 0.3 arcsecond using a closedloop servoactuated system. Nonsidereal rate tracking can be used, see Figure 6.

4.2. Dome. Each telescope is housed in an automatic motorized dome with a window opening from $0^{\circ}$ to $90^{\circ}$, see Figure 7. The domes are manufactured by Baader Planetarium.

4.3. Camera. Each telescope is equipped with a $4008 \times 2672$ refrigerated CCD Finger Lakes with a pixel size of $9 \mu \mathrm{m}$ 


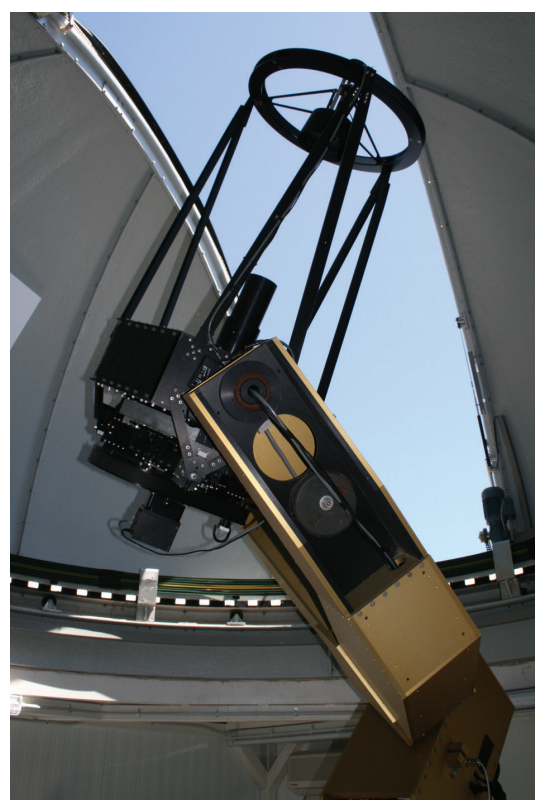

Figure 6: Complete view of the $0.5 \mathrm{~m}$ telescope inside the $3.5 \mathrm{~m}$ dome in Calar Alto Observatory.

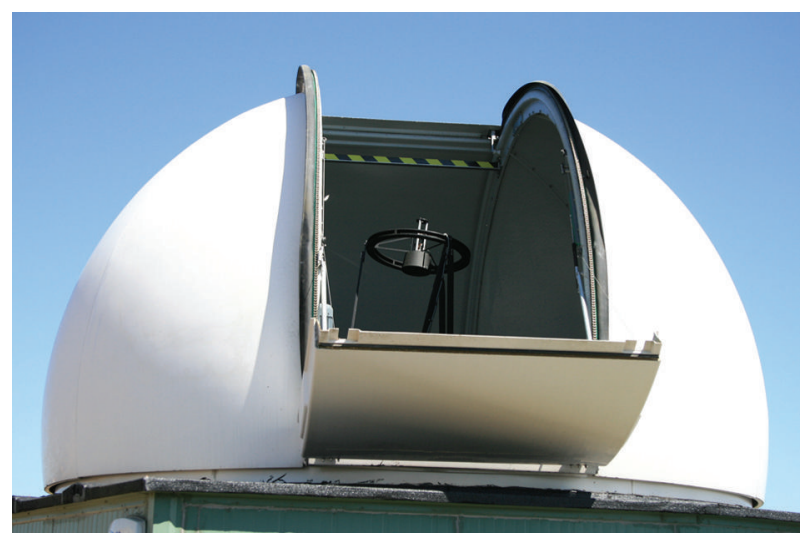

Figure 7: View of the dome for the $0.5 \mathrm{~m}$ telescope located in EVA1 El Frasno.

that covers a field of $24^{\prime} \times 16^{\prime}\left(30^{\prime} \times 20^{\prime}\right.$ in the case of INTA telescope) with a pixel projection of $0.37^{\prime \prime} / \mathrm{pix}$, see Figure 8. This is a cooled monochrome interline array manufactured by Kodak (KAI-11002) optimized for speed readout $(12 \mathrm{MHz})$ and a shutter capable of 0.05 -second exposures. There is also a filter wheel for each telescope with the typical UBVRI Johnson photometry set, nebula filters (OIII, $\mathrm{H} \alpha$, and $\mathrm{H} \beta$ ), and red, green, and blue filters.

4.4. Control System. The LINUX-based control system includes two computers per telescope, one dedicated to telescope control and the other for data analysis. There are some other facilities such as UPS, communications, GPS (for time synchronization), weather station, air conditioning to

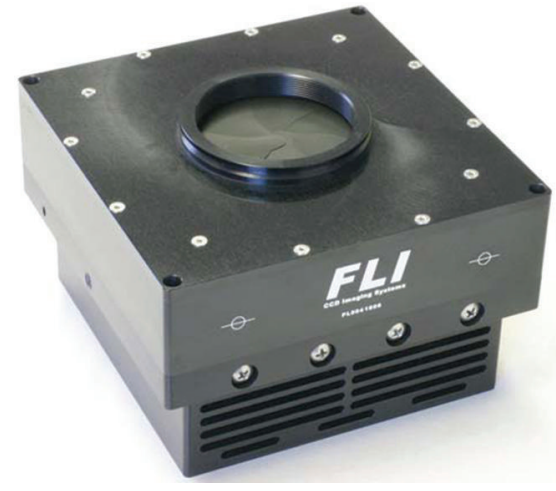

Figure 8: CCD Finger Lakes camera installed on the telescopes.

maintain the temperature inside the dome, and remote onoff system.

4.5. Control Programs. To provide complete control over the observatory, some other applications are also available, including sky programs that are used interactively with the telescopes and web cameras to view the status of the telescope and dome remotely.

4.6. Graphical User Interface. The observatories are controlled using the LINUX-based Talon software from Optical Mechanics, Inc. This application controls the entire observatory subsystems including the telescope and all its possible movements, the dome, the filter wheel, the temperaturecorrected focus, the weather station, and the CCD camera. There is a separate mode for data acquisition. Talon is operated by a very user friendly graphical user interface, see Figure 9.

4.7. Acquisition of Data. A specific application in Talon is dedicated to the acquisition of data. It controls the important camera settings, including the exposure time, the status of the shutter, the binning factor, subimages, and the temperature. Other tools including photometry, statistics, and a full-width half-maximum (FWHM) calculator are available.

4.8. Operation. There are three operation modes available:

(i) interactive and onsite, via the console,

(ii) interactive and remote via a login program,

(iii) noninteractive and robotic, with automatic preprogrammed tasks.

\section{Science with RTRCAB}

The main objective of this network is to detect new exoplanets, and especially to characterize the known exoplanets by observing photometric and systematic monitoring of their transits. The telescopes, equipped with advanced technology, optimized control programs, and optical and 


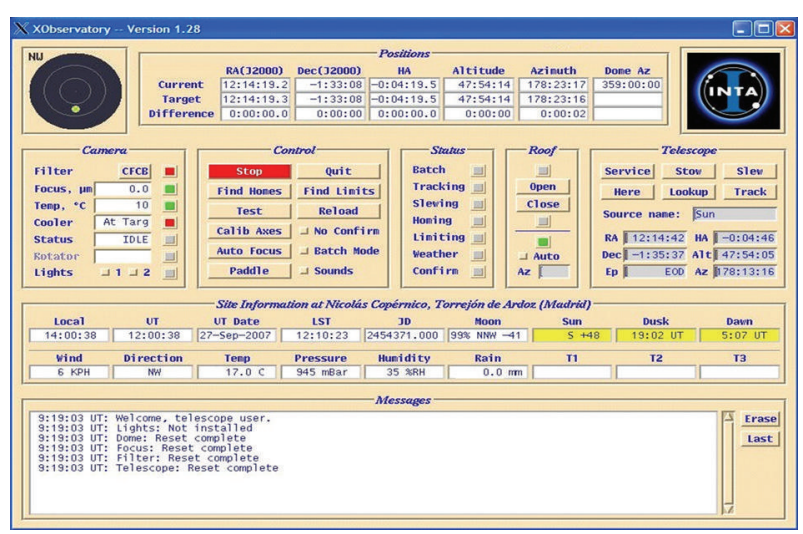

FIgURE 9: Talon graphical user interface for telescope control.

technical characteristics adequate for this purpose, are ideal to make the observations that are required to carry out these programs [21]. The achievement of these goals is ensured by the existence of three separated geographical stations.

But even if the first priority is the detection of exoplanets, a significant fraction of observing time will be available for other scientific projects of interest, objects relatively bright and variable, like Solar System objects, brown dwarfs, variable stars, planetary nebulae, and compact objects in binary systems.

5.1. Characterizing Exoplanets by the Transit Method. The idea of the depth of the project is the scientific exploitation of the RTRCAB from an astrobiological perspective. From this point of view, the detection of new exoplanets is the goal. The working method is to use the RTRCAB for monitoring beforehand selected stars by fast optical photometry. Usually we will work to obtain observational confirmation of the candidates from exoplanet hunter projects such as TrES or SuperWASP. However, we also work over our own select sky fields to find exoplanets. A previous work of selection may be done to know where to look for, the first stage with solar type stars and after that with M type stars or Planetary Nebulae.

Nearly continuous periods observing selected star are needed to try to find some changes in the light from the star. Comparison of photometry data from one night to another will be done to look for the slight variations that could indicate a possible transit. After that, the candidates will be reobserved to confirm results.

But not only is the search for exoplanets important but also the characterization is a goal. In this sense, we will develop a reliable database of parameters for exoplanets. The working method is similar to the previous goal. In this case we will observe stars with known transit exoplanets to obtain a light curve from which information about its parameters may be extracted. The repeated observation of these transits will substantially reduce the error bars and will provide a data set with accuracy so that they can be collated, thus allowing to draw conclusions about the nature of exoplanets and its formation conditions. Previously, we will select the exoplanets with more favorable conditions to be observed during their transits according to the characteristics of the telescopes: large size, low stellar temperature, low magnitude, and long transit. In this way we will restrict the sample to a particular group of exoplanets, which makes more sense to compare their parameters for their homogeneity.

Finally, the detection of the atmosphere in exoplanets is between our objectives. We will use again fast optical photometry during the transits of exoplanets to detect signs of its atmosphere by slight changes in the slope of entry and exit. Once the atmosphere is detected, we will develop geometric models to infer information about the atmosphere as size, opacity, and composition. We will also select those exoplanets with more favorable conditions for its atmosphere to be detected.

\section{Conclusions}

The RTRCAB (Red de Telescopios Robóticos del Centro de Astrobiología, Network of Robotic Telescopes at CAB) has three observation stations located in different sites of Spain: campus of INTA, in Torrejón de Ardoz (Madrid), Calar Alto Observatory (Almería), and in EVA1 in El Frasno, near Calatayud (Zaragoza). The network is designed for its use in scientific areas relevant to Astrobiology and, in turn, will support outreach programs and science education.

\section{References}

[1] D. Charbonneau, T. M. Brown, D. W. Latham, and M. Mayor, "Detection of planetary transits across a sun-like star," The Astrophysical Journal, vol. 529, no. 1, part 2, pp. L45-L48, 2000.

[2] M. Konacki, G. Torres, S. Jha, and D. D. Sasselov, "An extrasolar planet that transits the disk of its parent star," Nature, vol. 421, no. 6922, pp. 507-509, 2003.

[3] D. I. Steel and B. G. Marsden, "Astrometry of near-Earth objects using small telescopes," Earth, Moon and Planets, vol. 74, no. 2, pp. 85-92, 1996.

[4] P. R. Holvorcem, M. Schwartz, C. W. Juels, et al., "Astrometry of near-earth asteroids using remotely-operated robotic telescopes," in Astronomy in Latin America, 2nd Meeting on Astrometry in Latin America and 3rd Brazilian Meeting on Fundamental Astronomy, held on September 2002, R. Teixeira, N. V. Leister, V. A. F. Martin, and P. Benevides-Soares, Eds., vol. 1 of ADeLA Publications Series, pp. 91-100, 2003.

[5] M. Mayor and D. Queloz, "A search for substellar companions to solar-type stars via precise Doppler measurements: a first Jupiter mass companion detected," in Proceedings of the 9th Cambridge Workshop on Cool Stars, Stellar Systems and the Sun, vol. 109 of ASP Conference Series, pp. 35-38, 1996.

[6] M. Mayor and D. Queloz, "A Jupiter-mass companion to a solar-type star," Nature, vol. 378, no. 6555, pp. 355-359, 1995.

[7] G. W. Marcy and R. P. Butler, "A planetary companion to 70 virginis," The Astrophysical Journal, vol. 464, no. 2, part 2, pp. L147-L151, 1996.

[8] D. Charbonneau, T. M. Brown, R. W. Noyes, and R. L. Gilliland, "Detection of an extrasolar planet atmosphere," The Astrophysical Journal, vol. 568, no. 1, pp. 377-384, 2002.

[9] R. Alonso, M. Barbieri, M. Rabus, H. J. Deeg, J. A. Belmonte, and J. M. Almenara, "Limits to the planet candidate GJ 436c," Astronomy and Astrophysics, vol. 487, no. 1, pp. L5-L8, 2008. 
[10] G. Basri, “ Extrasolar planets: too close for comfort,” Nature, vol. 430, no. 6995, pp. 24-25, 2004.

[11] R. Alonso, T. M. Brown, G. Torres, et al., "TrES-1: the transiting planet of a bright $\mathrm{K} 0 \mathrm{~V}$ star," The Astrophysical Journal, vol. 613, no. 2, part 2, pp. L153-L156, 2004.

[12] D. Pollacco, I. Skillen, A. Collier Cameron, et al., "WASP3b: a strongly irradiated transiting gas-giant planet," Monthly Notices of the Royal Astronomical Society, vol. 385, no. 3, pp. 1576-1584, 2008.

[13] C. Moutou, H. Bruntt, T. Guillot, et al., “Transiting exoplanets from the CoRoT space mission: V. CoRoT-Exo-4b: stellar and planetary parameters," Astronomy and Astrophysics, vol. 488, no. 2, pp. L47-L50, 2008.

[14] G. Israellan, N. C. Santos, M. Mayor, and R. Rebolo, "Evidence for planet engulfment by the star HD82943," Nature, vol. 411, no. 6834 , pp. 163-166, 2001.

[15] A. Ecuvillon, G. Israelian, N. C. Santos, N. G. Shchukina, M. Mayor, and R. Rebolo, "Oxygen abundances in planetharbouring stars: comparison of different abundance indicators," Astronomy and Astrophysics, vol. 445, no. 2, pp. 633-645, 2006.

[16] C. J. Grillmair, A. Burrows, D. Charbonneau, et al., "Strong water absorption in the dayside emission spectrum of the planet HD 189733b," Nature, vol. 456, no. 7223, pp. 767-769, 2008.

[17] A. Vidal-Madjar, A. Lecaveller des Etangs, J.-M. Désert, et al., "An extended upper atmosphere around the extrasolar planet HD209458b," Nature, vol. 422, no. 6928, pp. 143-146, 2003.

[18] A. Vidal-Madjar, J.-M. Désert, A. Lecavelier Des Etangs, et al., "Detection of oxygen and carbon in the hydrodynamically escaping atmosphere of the extrasolar planet HD 209458B," The Astrophysical Journal, vol. 604, no. 1, part 2, pp. L69-L72, 2004.

[19] L. Cuesta, "A la búsqueda de exoplanetas," in Proceedings of the 8th Scientific Meeting of the Spanish Astronomical Society, Highlights of Spanish Astrophysics V, J. Gorgas, L. J. Goicoechea, J. I. González-Serrano, and J. M. Diego, Eds., in press.

[20] L. Cuesta, "Robotic telescopes and their use as an educational tool," in Proceedings of Communicating Astronomy with the Public, L. L. Christensen, M. Zoulais, and I. Robson, Eds., pp. 396-398, Athens, Greece, October 2007.

[21] L. Cuesta, "The network of INTA telescopes," in Proceedings of Communicating Astronomy with the Public, L. L. Christensen, M. Zoulais, and I. Robson, Eds., pp. 400-402, Athens, Greece, October 2007. 

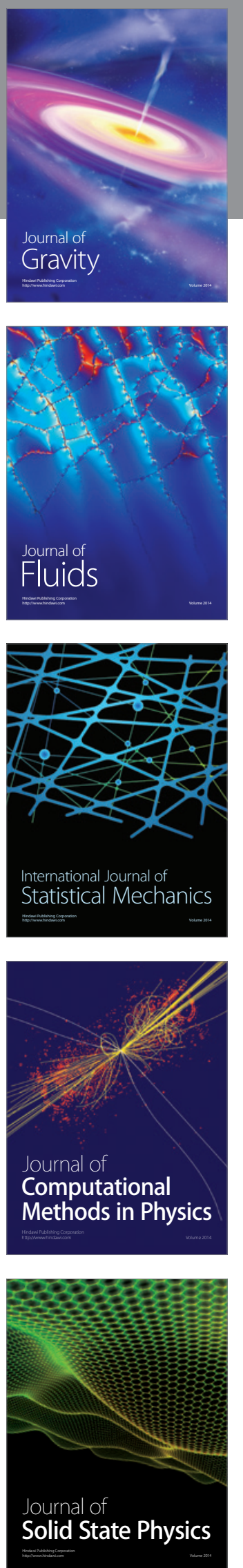

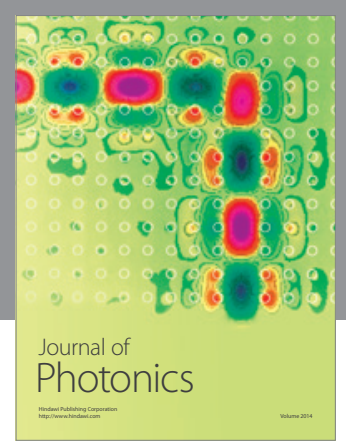

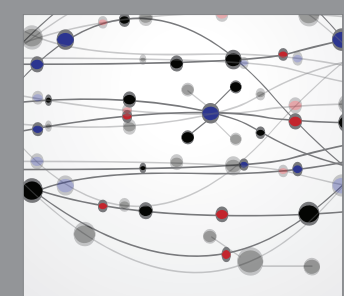

The Scientific World Journal
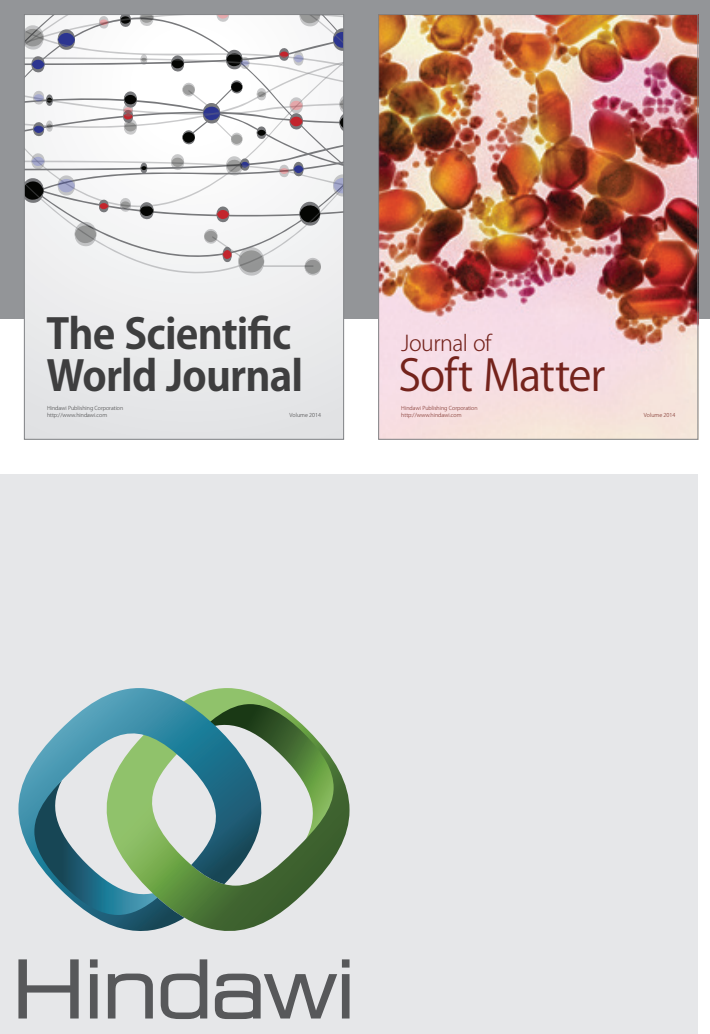

Submit your manuscripts at

http://www.hindawi.com
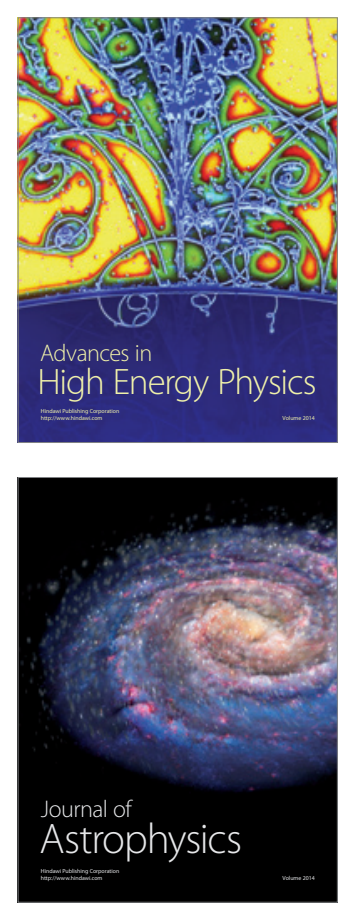
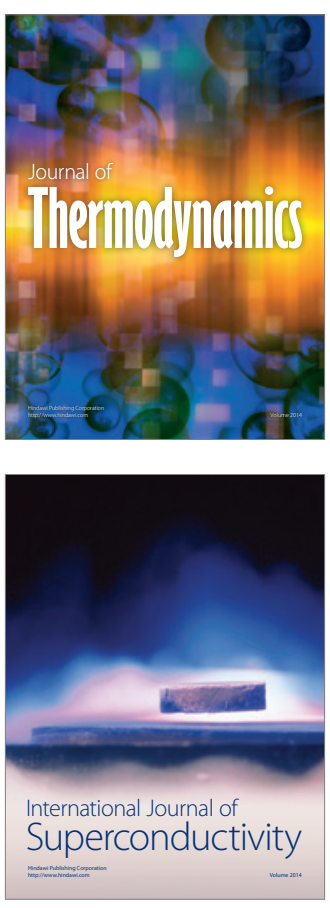
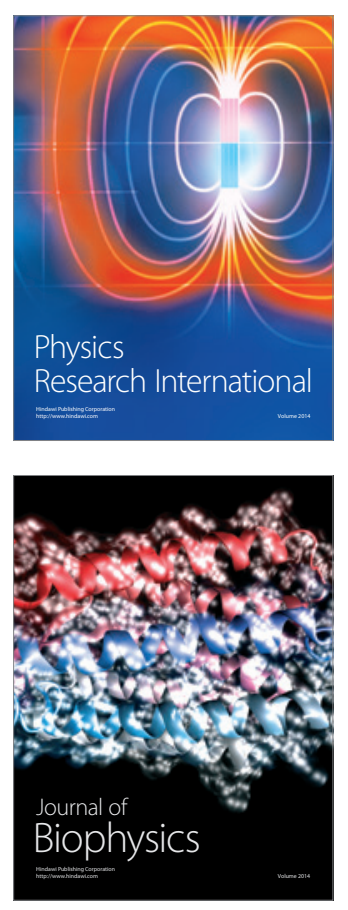
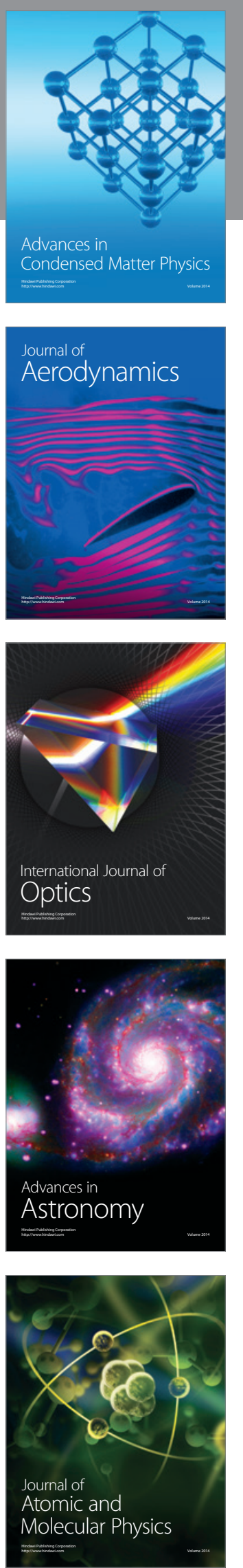